\title{
Middle cerebral artery aneurysms: aneurysm angiographic morphology and its relation to pre-operative and intra-operative rupture
}

\author{
Aneurismas da artéria cerebral média: morfologia angiográfica dos aneurismas e sua \\ relação com ruptura pré-operatória e intra-operatória \\ Iracema Araújo Estevão ${ }^{1}$, Bruno Camporeze', Antonio Santos de Araujo $\mathrm{Jr}^{2}$, Breno Nery³, \\ Ápio Claudio Martins Antunes ${ }^{4,5}$, Timothy R. Smith 6 , Paulo Henrique Pires de Aguiar ${ }^{3,5,7}$
}

\begin{abstract}
Objective: Correlate the middle cerebral artery bifurcation aneurysm morphology with the pre-operative and intra-operative risk of rupture. Methods: Forty patients with 46 middle cerebral artery bifurcation aneurysms were treated microsurgically by the same surgeon. Aneurysms were classified according to shape and the Fisher test was applied to analyze the effect of morphology on the pre-operative and intra-operative rupture. Results: Pre-operative and intra-operative ruptures were observed in 8/46 patients (17.4\%) and 14/46 patients (30.4\%) respectively. Thirty-two cases (69.6\%) had no symptoms postoperatively, modified Rankin score (MRS) of 0; 6.5\% had MRS of 1 (no significant disability); $13 \%$ had MRS of 2 (slight disability); $4.3 \%$ had moderately severe disability (MRS of 4); and there were 3 deaths (6.5\%) post-operatively. The morphology was not directly related to the rupture rate. Conclusion: In general, ruptures are not affected by the morphology or the studied variables. Larger series are needed to validate these outcomes.
\end{abstract}

Keywords: aneurysm; subarachnoid hemorrhage; middle cerebral artery.

RESUMO

Objetivo: Correlacionar a morfologia do aneurisma da bifurcação da artéria cerebral média com o risco de ruptura pré-operatória e intraoperatória. Métodos: 40 pacientes com 46 aneurismas de bifurcação da artéria cerebral média receberam tratamento microcirúrgico pelo mesmo cirurgião. Os aneurismas foram classificados de acordo com a morfologia e o teste de Fisher foi aplicado para analisar o efeito da morfologia sobre a ruptura pré-operatória e intra-operatória. Resultados: As rupturas pré e intra-operatória foram observadas em $8 / 46$ pacientes $(17,4 \%)$ e 14/46 (30,4\%) respectivamente. Trinta e dois casos (69,6\%) não apresentaram sintomas pós-operatórios, pontuação de Rankin modificada (MRS) de 0, 6,5\% tinham MRS de 1 (sem incapacidade significativa), 13\% tinham MRS de 2 (leve incapacidade), 4,3\% moderadamente grave (MRS de 4) e houve 3 óbitos (6,5\%) durante o pós-operatório. A morfologia não estava diretamente relacionada à taxa de ruptura pré-operatória ou intra-operatória. Conclusão: Em geral, as rupturas não são afetadas pela morfologia ou pelas variáveis estudadas. São necessárias séries maiores para validar esses resultados.

Palavras-chave: aneurisma; hemorragia subaracnoidea; artéria cerebral média.

Middle cerebral artery aneurysms are some of the most common vasculopathies in the anterior cerebral circulation, with an overall incidence of $18 \%$ to $20 \%$ of all aneurysms encountered ${ }^{1}$. Aneurysms in this location are typically complex, multi-lobed, and incorporate eloquent vascular branches; and therefore pose critical surgical management strategic points, particularly when they are ruptured. Aspects to be considered are shape, vascular variations (trifurcation, bifurcation, or anatomical variations such as middle cerebral artery duplication or “fenestration"), M1 segment length, size, branch

1 Universidade São Francisco, Bragança Paulista SP, Brasil;

${ }^{2}$ Araujo e Fazzito Neurocirurgião e Neurologistas Associados, Departamento de Neurocirurgia, São Paulo SP, Brasil;

${ }^{3}$ Hospital Santa Paula, Departamento de Neurocirurgia, São Paulo SP, Brasil;

${ }^{4}$ Hospital de Clínicas de Porto Alegre, Departamento de Neurocirurgia, Porto Alegre RS, Brasil;

${ }^{5}$ Universidade Federal do Rio Grande do Sul, Departamento de Neurocirurgia, Divisão de Pós-Graduação, Porto Alegre, Brasil;

${ }^{6}$ Harvard University, Department of Neurosurgery, Massachusetts, USA;

${ }^{7}$ Pontifícia Universidade Católica de São Paulo, Departamento de Neurologia, Sorocaba SP, Brasil.

Correspondence: Iracema Araújo Estevão; Rua Ercílio Baratella, 334, Edifício Mônica;12916-370 Bragança Paulista SP, Brasil;

E-mail:iaestevão@gmail.com

Conflict of interest: There is no conflict of interest to declare.

Received 20 January 2017; Accepted 30 March 2017. 
incorporation, and whether they are ruptured or not. The aneurysm neck is an important aspect when considering the endovascular treatment of the aneurysm. As opposed to endovascular therapies, neck-dome proportion is not as important when microsurgery is considered, since adequate neck dissection and sometimes aneurysm dome dissection are pivotal in determining complete aneurysm obliteration and avoidance of eloquent incorporated cortical branches. In order to obtain complete comprehension of the relationship between the nearby vascular branches and perforating arteries, surgical dissection of the neck and dome are sometimes needed when microsurgical clipping is considered; this is especially true when we are dealing with middle cerebral artery bifurcation aneurysm. The authors propose a simple morphological classification of middle cerebral artery bifurcation aneurysms in order to study the relationship of the aneurysm's morphology and its tendency to rupture pre-operatively or intra-operatively. The afore-mentioned morphological characteristics are particularly addressed by the authors, especially regarding the current technical repertoire and the surgeons' expertise.

\section{METHODS}

From January 1998 to July 2015, 40 patients with 46 bifurcation aneurysms of the middle cerebral artery were surgically clipped by the senior author. Pre-operative historical and radiological records were reviewed in detail and morphological aspects charted. Middle cerebral artery bifurcation aneurysms were classified according to shape into globoid, pear-shaped, bilobar, beak-shaped, multilobar, and fusiform (see classification model in Figure 1).

There were 27 females and 13 male patients. Average age was 50.20 years old $(\min =15$ years old, $\max =75$ years old $)$.

The same surgeon and surgical team operated all the aneurysms. Categorical and scalar variables were described for each patient and Fisher's test was applied to analyze the effect of the morphology on the pre-operative and intra-operative rupture rate. Fisher's test was also applied to verify the degree of association between the interest categorical variables (right side, left side, gender, presence of associated aneurysms other than middle cerebral artery bifurcation aneurysms, anatomical features such as an M1 segment of the middle cerebral artery larger than $3 \mathrm{~cm}$, presence of trifurcated middle cerebral artery and aspect ratio higher than 1.5) and the rupture variables. The Mann-Whitney test was applied to verify the degree of association between the scalar variables (age and size of the aneurysm) and the rupture variables.

\section{Classification}

We classified middle cerebral artery aneurysms based on their shapes into globoid, pear-shaped, beak-shaped, bilobar, multilobar and associated with fibromuscular dysplasia ( fusiform), as shown in Figure 1.

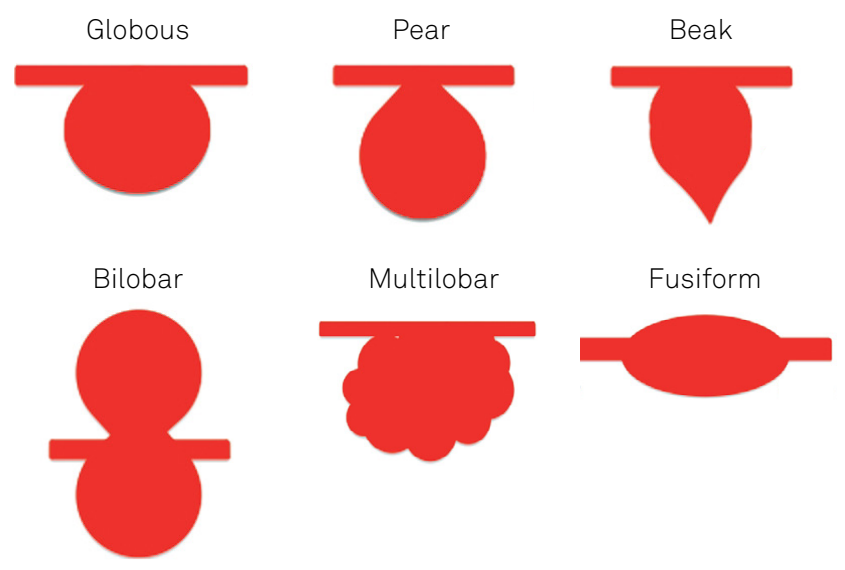

Figure 1. Classification model of aneurysms according to shape into globoid, pear -shaped, bilobar, beak-shaped, multilobar and fusiform.

There were 16 beak-shaped aneurysms (34.8\% of all aneurysms) with one pre-operative rupture in this category. Pearshaped aneurysms were also seen in 14 of the aneurysms (30.4\%), with one ruptured aneurysm at presentation. A globous shape was encountered in nine cases $(19.60 \%$ of the aneurysms), with one ruptured aneurysm at presentation (11.1\%). A bilobar shape was seen in two cases (4.3\%) with one ruptured aneurysm at presentation (50\%). A multilobar shape was seen in three cases (6.5\%) with two ruptured aneurysms at presentation (66.6\%). A fusiform aneurysm was seen in two cases $(4.3 \%)$ and none of those were ruptured at presentation. All the categorical variables evaluated during data collection are described in Table 1. Examples of the morphology can be seen in Figures 2 and 3 (A to E).

\section{Middle cerebral artery segment anatomic variations}

A long M1 segment was observed in 26\%, M1 trifurcation was observed in approximately $11 \%$, and M1 bifurcation was observed in $89 \%$ of the angiographies.

\section{Associated aneurysms}

Seven cases had one associated aneurysm other than the bifurcation of the middle cerebral artery on the right side and two patients had two associated aneurysms on the right side of the head. Four patients had one associated aneurysm on the left side other than the bifurcation of the middle cerebral artery (Table 1). Only one aneurysm was distal to the bifurcation of the middle cerebral artery. All the ruptures were related to the aneurysm of the bifurcation of the middle cerebral artery. Spearman's analysis was done to verify the degree of relationship between the variables of interest (Table 2). The relationship between the presence of associated aneurysms and middle cerebral artery bifurcation aneurysms, as well as the relationship between left side and right side of the associated aneurysms are weak; that is, nothing makes us believe that there is a higher probability of finding an associated rupture due to previously-ruptured aneurysms in our series (Table 2). 
Table 1. Description of all the categorical variables evaluated during data collection.

\begin{tabular}{|c|c|c|c|}
\hline Variable & Category & Frequency & Percentage \\
\hline \multirow{2}{*}{ Pre-operative rupture } & absent & 38 & 82.6 \\
\hline & present & 8 & 17.4 \\
\hline \multirow{2}{*}{ Right sided middle cerebral artery bifurcation aneurysm } & absent & 23 & 50 \\
\hline & present & 23 & 50 \\
\hline \multirow{2}{*}{ Left sided middle cerebral artery bifurcation aneurysm } & absent & 23 & 50 \\
\hline & present & 23 & 50 \\
\hline \multirow{13}{*}{ Year of surgery } & 1998 & 4 & 8.7 \\
\hline & 1999 & 1 & 2.2 \\
\hline & 2005 & 1 & 2.2 \\
\hline & 2006 & 3 & 6.5 \\
\hline & 2007 & 2 & 4.3 \\
\hline & 2008 & 8 & 17.4 \\
\hline & 2009 & 6 & 13 \\
\hline & 2010 & 2 & 4.3 \\
\hline & 2011 & 2 & 4.3 \\
\hline & 2012 & 5 & 10.9 \\
\hline & 2013 & 5 & 10.9 \\
\hline & 2014 & 2 & 4.3 \\
\hline & 2015 & 5 & 10.9 \\
\hline \multirow{2}{*}{ Sex } & female & 32 & 69.6 \\
\hline & male & 14 & 30.4 \\
\hline \multirow{2}{*}{ Intra-operative rupture } & absent & 32 & 69.6 \\
\hline & Present & 14 & 30.4 \\
\hline \multirow{5}{*}{ Modified rankin score } & 0 & 32 & 69.6 \\
\hline & 1 & 3 & 6.5 \\
\hline & 2 & 6 & 13 \\
\hline & 4 & 2 & 4.3 \\
\hline & 6 & 3 & 6.5 \\
\hline \multirow{4}{*}{ Presence of associated right side aneurysms other than the bifurcation of the MCA } & 0 & 37 & 80.4 \\
\hline & 1 & 7 & 15.2 \\
\hline & 2 & 2 & 4.3 \\
\hline & $>2$ & 0 & 0.0 \\
\hline \multirow{4}{*}{ Presence of associated Left Side Aneurysms other than the bifurcation of the MCA } & 0 & 42 & 91.3 \\
\hline & 1 & 4 & 8.7 \\
\hline & 2 & 0 & 0.0 \\
\hline & $>2$ & 0 & 0.0 \\
\hline \multirow{2}{*}{ Pear-shaped middle cerebral artery aneurysm } & Absent & 32 & 69.6 \\
\hline & Present & 14 & 30.4 \\
\hline \multirow{2}{*}{ Beak-shaped middle cerebral artery aneurysm } & Absent & 30 & 65.2 \\
\hline & Present & 16 & 34.8 \\
\hline \multirow{2}{*}{ Globous-shaped middle cerebral artery aneurysm } & Absent & 37 & 80.4 \\
\hline & Present & 9 & 19.6 \\
\hline \multirow{2}{*}{ Bilobar-shaped middle cerebral artery aneurysm } & Absent & 44 & 95.7 \\
\hline & Present & 2 & 4.3 \\
\hline \multirow{2}{*}{ Multilobar-shaped middle cerebral artery aneurysm } & Absent & 43 & 93.5 \\
\hline & Present & 3 & 6.5 \\
\hline \multirow{2}{*}{ Fusiform-shaped middle cerebral artery aneurysm } & Absent & 44 & 95.7 \\
\hline & Present & 2 & 4.3 \\
\hline \multirow{2}{*}{ M1 segment longer than $3 \mathrm{~cm}$} & Absent & 34 & 73.9 \\
\hline & Present & 12 & 26.1 \\
\hline \multirow{2}{*}{ Trifurcated middle cerebral artery } & Absent & 41 & 89.1 \\
\hline & Present & 5 & 10.9 \\
\hline \multirow{2}{*}{ Aspect ratio $>1.5$} & Absent & 18 & 39.1 \\
\hline & Present & 28 & 60.9 \\
\hline
\end{tabular}

MCA: Middle Cerebral Artery; M1: Segment M1 of Middle Cerebral Artery. 

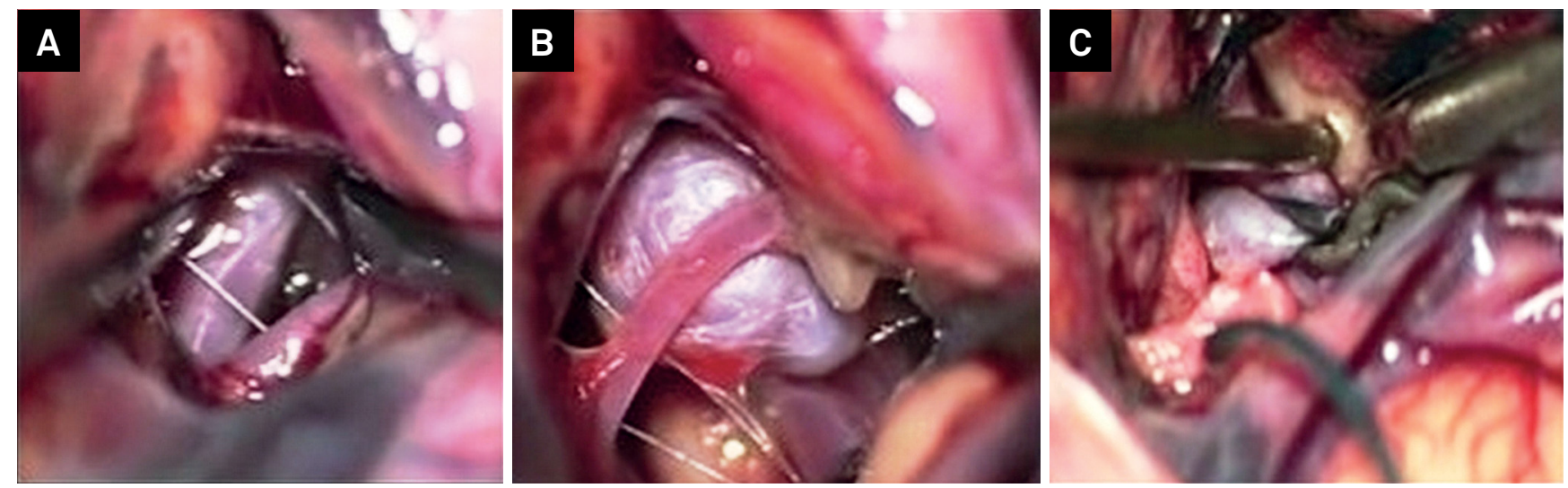

Figure 2. Intra-operative vision and clipping of middle cerebral artery aneurysms.
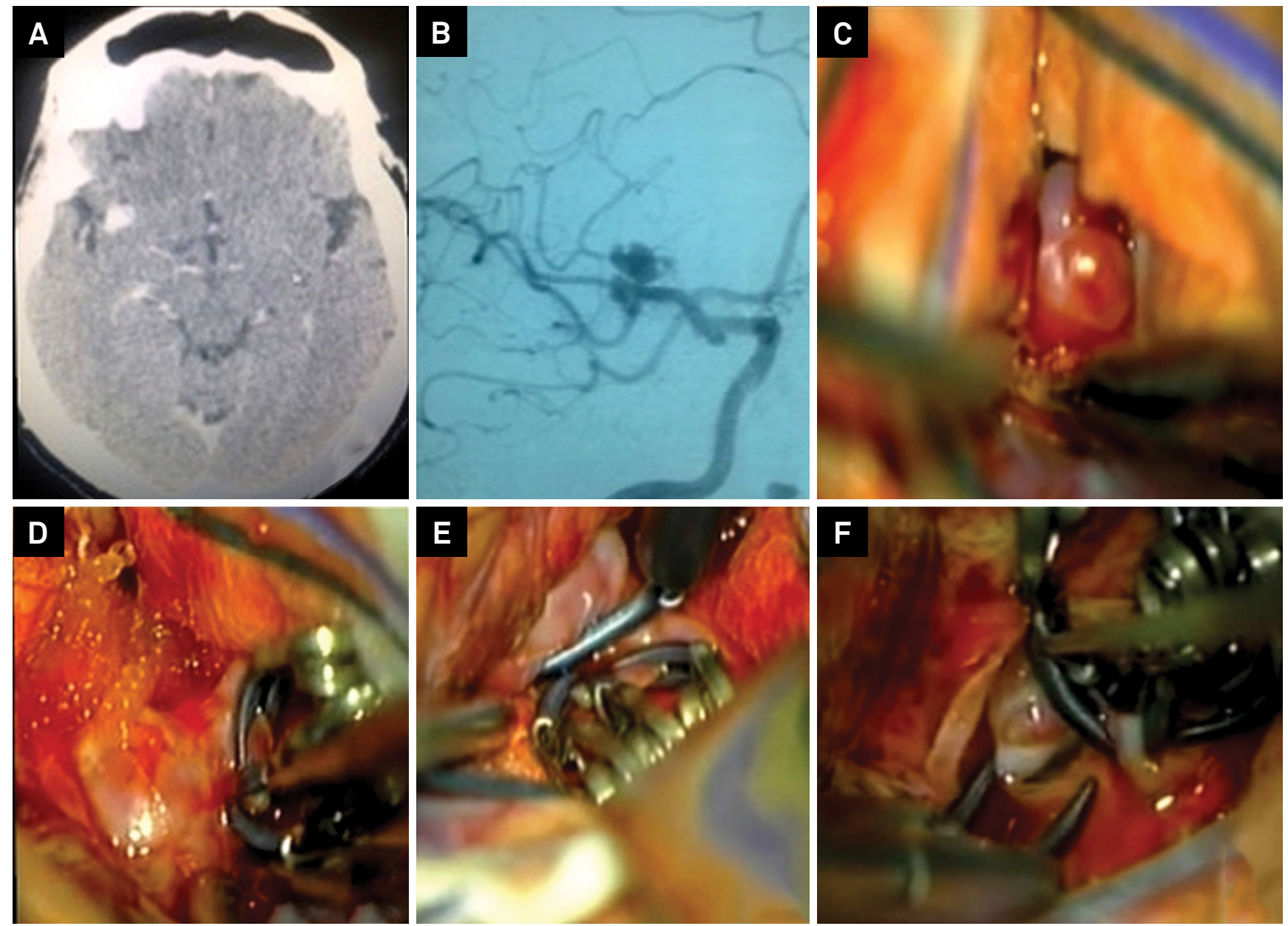

Figure 3. A. CT scan axial section of a patient with middle cerebral artery aneurysm. B. Angiogram of a patient with MCA aneurysm in M2 portion. C. Identification of the aneurysm. D. E. F. Intra-operative vision of clipping of a middle cerebral artery aneurysm.

\section{RESULTS}

The average aneurysm size was $6.59 \mathrm{~mm}$. Pre-operative and intra-operative ruptures were observed in $8 / 46$ patients (17.4\%) and $14 / 46$ patients $(30.4 \%)$ respectively. The average age was 50.2 years old (15-75), with a median age of 51.5 years of age (Table 3). Thirty-two cases (69.6\%) had no symptoms postoperatively (modified Rankin score (MRS) of 0, 6.5\% had an MRS of 1 (no significant disability), 13\% had MRS of 2 (slight disability), $4.30 \%$ had moderately severe disability (MRS of 4 ) and there were 3 deaths $(6.5 \%)$ post-operatively. Fourteen aneurysms were classified as pear shaped (30.4\%), 16 aneurysms were classified as beak shaped (34.8\%), 9 were considered to be globous shaped (19.6\%), 2 patients (4.3\%) had bilobar shaped aneurysms, 3 patients (6.5\%) had multilobar shaped aneurysms and 2 (4.3\%) had fusiform aneurysms (Table 1 ). 
Fisher's test was applied to analyze the effect of morphology on the pre-operative and intra-operative rupture (Tables 4 and 5, respectively). Statistical analysis showed that the morphology of the aneurysm does not affect the rate of pre-operative or intra-operative rupture. Fisher's test was also applied to verify the degree of association between the categorical variables of interest (right side, left side, gender, presence of associated aneurysms other than middle cerebral artery bifurcation aneurysms, anatomical features such as an M1 segment of the middle cerebral artery larger than $3 \mathrm{~cm}$, the presence of a trifurcated middle cerebral artery and aspect ratio higher than 1.5) and the rupture variables
(Tables 6 and 7). The Mann-Whitney test was applied to verify the degree of association between the scalar variables (age and size of the aneurysm) and the rupture variables (Tables 8 and 9). According to the analyses, none of the variables seemed to affect the rate of pre-operative or intra-operative rupture. Spearman's correlation analysis was applied to verify the degree of relationship between the morphology variables and the above-mentioned categorical variables of interest (Table 10). According to this analysis the relationship between those variables seemed to be weak, showing that they do not represent bias when correlating the morphology of the aneurysms and the risk of rupture.

Table 2. Spearman analysis was done to verify the degree of relationship between the variables of interest.

\begin{tabular}{|c|c|c|c|c|}
\hline Variable & Statistics & $\begin{array}{l}\text { Right sided bifurcation } \\
\text { middle cerebral artery } \\
\text { aneurysm }\end{array}$ & $\begin{array}{l}\text { Left sided bifurcation } \\
\text { middle cerebral artery } \\
\text { aneurysm }\end{array}$ & $\begin{array}{c}\text { Presence of associated } \\
\text { left side aneurysms other } \\
\text { than the bifurcation of } \\
\text { the MCA }\end{array}$ \\
\hline \multirow{3}{*}{$\begin{array}{l}\text { Left sided middle } \\
\text { cerebral artery } \\
\text { bifurcation aneurysm }\end{array}$} & Coef. Correl. (r) & -1.000 & - & - \\
\hline & Sig. (p) & $<0.001$ & - & - \\
\hline & $n$ & 46 & - & - \\
\hline \multirow{3}{*}{$\begin{array}{l}\text { Right sided middle } \\
\text { cerebral artery } \\
\text { bifurcation aneurysm }\end{array}$} & Coef. Correl. (r) & +0.031 & -0.031 & - \\
\hline & Sig. (p) & 0.839 & 0.839 & - \\
\hline & $n$ & 46 & 46 & - \\
\hline \multirow{3}{*}{$\begin{array}{l}\text { Presence of associated } \\
\text { left sided aneurysms } \\
\text { other than the bifurcation } \\
\text { of the MCA }\end{array}$} & Coef. Correl. (r) & 0.000 & 0.000 & -0.152 \\
\hline & Sig. (p) & $>0.999$ & $>0.999$ & 0.315 \\
\hline & $n$ & 46 & 46 & 46 \\
\hline
\end{tabular}

MCA: Middle Cerebral Artery; Coef. Correl.: Correlation Coeffcient ; Sig: statistical significance.

Table 3. Scalar variables (age and size of the operated aneurysms).

\begin{tabular}{lccccccc} 
Variable & $\mathrm{n}$ & Min & Max & Average & $\begin{array}{c}\text { Standard } \\
\text { deviation }\end{array}$ & $\begin{array}{r}\text { Percentile 25 } \\
\begin{array}{c}\text { Percentile } 50 \\
\text { (Median) }\end{array}\end{array}$ Percentile 75 \\
Age & 46 & 15 & 75 & 50.2 & 12.9 & 42 & 51.5 \\
Size of the aneurysm & 46 & 3 & 15 & 6.59 & 2.65 & 5 & 6.75 \\
\hline
\end{tabular}

Table 4. Fisher's test to verify the association between the morphology of the aneurysms and the pre-operative rate of rupture.

\begin{tabular}{|c|c|c|c|c|c|c|}
\hline \multirow{3}{*}{$\begin{array}{l}\text { Middle cerebral artery } \\
\text { aneurysm }\end{array}$} & \multirow{3}{*}{ Category } & \multicolumn{4}{|c|}{ Pre-operative Rupture } & \multirow{3}{*}{ Sig. (p) } \\
\hline & & \multicolumn{2}{|c|}{ Absent } & \multicolumn{2}{|c|}{ Present } & \\
\hline & & Frequency & Percentage & Frequency & Percentage & \\
\hline \multirow{2}{*}{ Pear-shaped } & Absent & 25 & 65.8 & 7 & 87.5 & \multirow{2}{*}{0.225} \\
\hline & Present & 13 & 34.2 & 1 & 12.5 & \\
\hline \multirow{2}{*}{ Beak-shaped } & Absent & 24 & 63.2 & 6 & 75 & \multirow{2}{*}{0.523} \\
\hline & Present & 14 & 36.8 & 2 & 25 & \\
\hline \multirow{2}{*}{ Globous-shaped } & Absent & 31 & 81.6 & 6 & 75 & \multirow{2}{*}{0.670} \\
\hline & Present & 7 & 18.4 & 2 & 25 & \\
\hline \multirow{2}{*}{ Bilobar-shaped } & Absent & 37 & 97.4 & 7 & 87.5 & \multirow{2}{*}{0.213} \\
\hline & Present & 1 & 2.6 & 1 & 12.5 & \\
\hline \multirow{2}{*}{ Multilobar-shaped } & Absent & 37 & 97.4 & 6 & 75 & \multirow{2}{*}{0.020} \\
\hline & Present & 1 & 2.6 & 2 & 25 & \\
\hline \multirow{2}{*}{ Fusiform-shaped } & Absent & 36 & 94.7 & 8 & 100 & \multirow{2}{*}{0.507} \\
\hline & Present & 2 & 5.3 & 0 & 0.0 & \\
\hline
\end{tabular}


Table 5. Fisher's test to verify the association between the morphology of the aneurysms and the intra-operative rate of rupture.

\begin{tabular}{|c|c|c|c|c|c|c|}
\hline \multirow{3}{*}{ Middle cerebral artery aneurysm } & \multirow{3}{*}{ Category } & \multicolumn{4}{|c|}{ Intra-operative rupture } & \multirow{3}{*}{ Sig. (p) } \\
\hline & & \multicolumn{2}{|c|}{ Absent } & \multicolumn{2}{|c|}{ Present } & \\
\hline & & Frequency & Percentage & Frequency & Percentage & \\
\hline \multirow{2}{*}{ Pear-shaped } & Absent & 24 & 75 & 8 & 57.1 & \multirow{2}{*}{0.226} \\
\hline & Present & 8 & 25 & 6 & 42.9 & \\
\hline \multirow{2}{*}{ Beak-shaped } & Absent & 19 & 59.4 & 11 & 78.6 & \multirow{2}{*}{0.208} \\
\hline & Present & 13 & 40.6 & 3 & 21.4 & \\
\hline \multirow{2}{*}{ Globous-shaped } & Absent & 27 & 84.4 & 10 & 71.4 & \multirow{2}{*}{0.308} \\
\hline & Present & 5 & 15.6 & 4 & 28.6 & \\
\hline \multirow{2}{*}{ Bilobar-shaped } & Absent & 30 & 93.8 & 14 & 100 & \multirow{2}{*}{0.339} \\
\hline & Present & 2 & 6.3 & 0 & 0.0 & \\
\hline \multirow{2}{*}{ Multilobar-shaped } & Absent & 30 & 93.8 & 13 & 92.9 & \multirow{2}{*}{0.910} \\
\hline & Present & 2 & 6.3 & 1 & 7.1 & \\
\hline \multirow{2}{*}{ Fusifor-shaped } & Absent & 30 & 93.8 & 14 & 100 & \multirow{2}{*}{0.339} \\
\hline & Present & 2 & 6.3 & 0 & 0.0 & \\
\hline
\end{tabular}

Table 6. Fisher's test to verify the association between the variables of interest and pre-operative rupture.

\begin{tabular}{|c|c|c|c|c|c|c|}
\hline \multirow{3}{*}{ Variable } & \multirow{3}{*}{ Category } & \multicolumn{4}{|c|}{ Pre-operative Rupture } & \multirow{3}{*}{ Sig. (p) } \\
\hline & & \multicolumn{2}{|c|}{ Absent } & \multicolumn{2}{|c|}{ Present } & \\
\hline & & Frequency & Percentage & Frequency & Percentage & \\
\hline \multirow{2}{*}{$\begin{array}{l}\text { Right sided middle cerebral artery } \\
\text { bifurcation aneurysm }\end{array}$} & Absent & 19 & 50 & 4 & 50 & \multirow{2}{*}{$>0.999$} \\
\hline & Present & 19 & 50 & 4 & 50 & \\
\hline \multirow{2}{*}{$\begin{array}{l}\text { Left sided middle cerebral artery } \\
\text { bifurcation aneurysm }\end{array}$} & Absent & 19 & 50 & 4 & 50 & \multirow{2}{*}{$>0.999$} \\
\hline & Present & 19 & 50 & 4 & 50 & \\
\hline \multirow{2}{*}{ Sex } & Female & 26 & 68.4 & 6 & 75 & \multirow{2}{*}{0.713} \\
\hline & Male & 12 & 31.6 & 2 & 25 & \\
\hline \multirow{4}{*}{$\begin{array}{l}\text { Presence of associated right } \\
\text { side aneurysms other than the } \\
\text { bifurcation of the MCA }\end{array}$} & 0 & 29 & 76.3 & 8 & 100 & \multirow{4}{*}{0.308} \\
\hline & 1 & 7 & 18.4 & 0 & 0.0 & \\
\hline & 2 & 2 & 5.3 & 0 & 0.0 & \\
\hline & $>2$ & 0 & $0, .0$ & 0 & 0.0 & \\
\hline \multirow{4}{*}{$\begin{array}{l}\text { Presence of associated left } \\
\text { side aneurysms other than the } \\
\text { bifurcation of the MCA }\end{array}$} & 0 & 34 & 89.5 & 8 & 100 & \multirow{4}{*}{0.337} \\
\hline & 1 & 4 & 10.5 & 0 & 0.0 & \\
\hline & 2 & 0 & 0.0 & 0 & 0.0 & \\
\hline & $>2$ & 0 & 0.0 & 0 & 0.0 & \\
\hline \multirow{2}{*}{ M1 segment $>3 \mathrm{~cm}$} & Absent & 29 & 76.3 & 5 & 62.5 & \multirow{2}{*}{0.419} \\
\hline & Present & 9 & 23.7 & 3 & 37.5 & \\
\hline \multirow{2}{*}{ Trifurcated middle cerebral artery } & Absent & 34 & 89.5 & 7 & 87.5 & \multirow[b]{2}{*}{0.871} \\
\hline & Present & 4 & 105 & 1 & 125 & \\
\hline \multirow{3}{*}{ Aspect ratio $>1.5$} & Absent & 15 & 39.5 & 3 & 37.5 & \multirow{3}{*}{0.917} \\
\hline & & & & & & \\
\hline & Present & 23 & 60.5 & 5 & 62.5 & \\
\hline
\end{tabular}

MCA: Middle Cerebral Artery ;M1: Segment M1 of Middle Cerebral Artery. 


\begin{tabular}{|c|c|c|c|c|c|c|}
\hline \multirow{3}{*}{ Variable } & \multirow{3}{*}{ Category } & \multicolumn{4}{|c|}{ Pre-operative Rupture } & \multirow{3}{*}{ Sig. (p) } \\
\hline & & \multicolumn{2}{|c|}{ Absent } & \multicolumn{2}{|c|}{ Present } & \\
\hline & & Frequency & Percentage & Frequency & Percentage & \\
\hline \multirow{2}{*}{$\begin{array}{l}\text { Right sided middle cerebral artery } \\
\text { bifurcation aneurysm }\end{array}$} & Absent & 19 & 50 & 4 & 50 & \multirow{2}{*}{$>0.999$} \\
\hline & Present & 19 & 50 & 4 & 50 & \\
\hline \multirow{2}{*}{$\begin{array}{l}\text { Left sided middle cerebral artery } \\
\text { bifurcation aneurysm }\end{array}$} & Absent & 19 & 50 & 4 & 50 & \multirow{2}{*}{$>0.999$} \\
\hline & Present & 19 & 50 & 4 & 50 & \\
\hline \multirow{3}{*}{ Sex } & Female & 26 & 68.4 & 6 & 75 & \multirow{3}{*}{0.713} \\
\hline & & & & & & \\
\hline & Male & 12 & 31.6 & 2 & 25 & \\
\hline \multirow{4}{*}{$\begin{array}{l}\text { Presence of associated right } \\
\text { side aneurysms other than the } \\
\text { bifurcation of the MCA }\end{array}$} & 0 & 29 & 76.3 & 8 & 100 & \multirow{4}{*}{0.308} \\
\hline & 1 & 7 & 18.4 & 0 & 0.0 & \\
\hline & 2 & 2 & 5.3 & 0 & 0.0 & \\
\hline & $>2$ & 0 & $0, .0$ & 0 & 0.0 & \\
\hline \multirow{4}{*}{$\begin{array}{l}\text { Presence of associated left } \\
\text { side aneurysms other than the } \\
\text { bifurcation of the MCA }\end{array}$} & 0 & 34 & 89.5 & 8 & 100 & \multirow{4}{*}{0.337} \\
\hline & 1 & 4 & 10.5 & 0 & 0.0 & \\
\hline & 2 & 0 & 0.0 & 0 & 0.0 & \\
\hline & $>2$ & 0 & 0.0 & 0 & 0.0 & \\
\hline \multirow{3}{*}{ M1 segment $>3 \mathrm{~cm}$} & Absent & 29 & 76.3 & 5 & 62.5 & \multirow{3}{*}{0.419} \\
\hline & & & & & & \\
\hline & Present & 9 & 23.7 & 3 & 37.5 & \\
\hline \multirow{3}{*}{ Trifurcated middle cerebral artery } & Absent & 34 & 89.5 & 7 & 87.5 & \multirow{3}{*}{0.871} \\
\hline & Drocent & 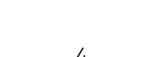 & 10 & 1 & 10 & \\
\hline & & & & & & \\
\hline \multirow{3}{*}{ Aspect ratio $>1.5$} & Absent & 15 & 39.5 & 3 & 37.5 & \multirow{3}{*}{0.917} \\
\hline & & & & & & \\
\hline & Present & 23 & 60.5 & 5 & 62.5 & \\
\hline
\end{tabular}

MCA: Middle Cerebral Artery; M1: Segment M1 of Middle Cerebral Artery.

Table 8. Mann-Whitney's test was applied to verify the degree of association between the scalar variables (age and size of the aneurysm) and pre-operative rupture.

\begin{tabular}{|c|c|c|c|c|c|c|c|c|c|c|}
\hline Variable & $\begin{array}{l}\text { Pre-operative } \\
\text { rupture }\end{array}$ & $n$ & Mean & SD & Min & Max & Percentile 25 & $\begin{array}{l}\text { Percentile } 50 \\
\text { (Median) }\end{array}$ & Percentile 75 & $\begin{array}{l}\text { Sig. } \\
\text { (p) }\end{array}$ \\
\hline \multirow{3}{*}{ Age } & Absent & 38 & 50.76 & 13.46 & 15 & 75 & 42.75 & 52.00 & 62.00 & \multirow{3}{*}{0.303} \\
\hline & Present & 8 & 47.50 & 10.09 & 32 & 65 & 39.5 & 49.50 & 52.50 & \\
\hline & Total & 46 & 50.20 & 12.90 & 15 & 75 & 42 & 51.50 & 59.75 & \\
\hline \multirow{3}{*}{$\begin{array}{l}\text { Size of the } \\
\text { aneurysm }\end{array}$} & Absent & 38 & 6.37 & 2.57 & 3 & 15 & 5 & 6.00 & 7.00 & \multirow{3}{*}{0.209} \\
\hline & Present & 8 & 7.63 & 2.93 & 5 & 12 & 5 & 7.00 & 11.00 & \\
\hline & Total & 46 & 6.59 & 2.65 & 3 & 15 & 5 & 6.00 & 7.25 & \\
\hline
\end{tabular}

SD: standard deviation. 
Table 9. Mann-Whitney's test applied to verify the degree of association between the scalar variables (age and size of the aneurysm) and intra-operative rupture.

\begin{tabular}{|c|c|c|c|c|c|c|c|c|c|c|}
\hline Variable & $\begin{array}{l}\text { Intra-operative } \\
\text { Rupture }\end{array}$ & $\mathrm{n}$ & Mean & SD & Min & Max & $\begin{array}{l}\text { Percentile } \\
\quad 25\end{array}$ & $\begin{array}{c}\text { Percentile } \\
50 \text { (Median) }\end{array}$ & $\begin{array}{l}\text { Percentile } \\
\quad 75\end{array}$ & Sig. (p) \\
\hline \multirow{3}{*}{ Age } & Absent & 32 & 51.34 & 12.55 & 15 & 75 & 44.5 & 52 & 58.75 & \multirow{3}{*}{0.2624} \\
\hline & Present & 14 & 47.57 & 13.78 & 21 & 67 & 38.75 & 46.5 & 62.75 & \\
\hline & Total & 46 & 50.2 & 12.9 & 15 & 75 & 42 & 51.5 & 59.75 & \\
\hline \multirow{3}{*}{$\begin{array}{l}\text { Size of the } \\
\text { aneurysm }\end{array}$} & Absent & 32 & 6.63 & 2.94 & 3 & 15 & 5 & 6 & 8 & \multirow{3}{*}{0.618} \\
\hline & Present & 14 & 6.5 & 1.91 & 5 & 12 & 5 & 6.5 & 7 & \\
\hline & Total & 46 & 6.59 & 2.65 & 3 & 15 & 5 & 6 & 7.25 & \\
\hline
\end{tabular}

SD: standard deviation.

Table 10. Spearman correlation analysis to verify the degree of relationship between the morphology variables (first line above) and the categorical variables of interest (left column).

\begin{tabular}{|c|c|c|c|c|c|c|c|}
\hline Variable & Statistics & Pear* & Beak* & Globous* & Bilobar* & Multilobar* & Fusiform* \\
\hline \multirow{3}{*}{$\begin{array}{l}\text { Right sided middle cerebral artery } \\
\text { bifurcation aneurysm }\end{array}$} & Coef. Correl. (r) & 0.000 & 0.365 & -0.164 & 0.000 & -0.264 & -0.213 \\
\hline & Sig. (p) & $>0.999$ & 0,013 & 0.275 & $>0.999$ & 0.076 & 0.155 \\
\hline & $n$ & 46 & 46 & 46 & 46 & 46 & 46 \\
\hline \multirow{3}{*}{$\begin{array}{l}\text { Left sided middle cerebral artery } \\
\text { bifurcation aneurysm }\end{array}$} & Coef. Correl. (r) & 0.000 & -0.365 & 0.164 & 0.000 & 0.264 & 0.213 \\
\hline & Sig. (p) & $>0.999$ & 0.013 & 0.275 & $>0.999$ & 0.076 & 0.155 \\
\hline & $n$ & 46 & 46 & 46 & 46 & 46 & 46 \\
\hline \multirow{3}{*}{ Age } & Coef. Correl. (r) & 0.153 & 0.002 & -0.008 & 0.088 & -0.100 & -0.301 \\
\hline & Sig. (p) & 0.310 & 0.991 & 0.957 & 0.559 & 0.510 & 0.042 \\
\hline & $n$ & 46 & 46 & 46 & 46 & 46 & 46 \\
\hline \multirow{3}{*}{ Sex } & Coef. Correl. (r) & 0.076 & -0.086 & -0.088 & 0.091 & 0.017 & 0.091 \\
\hline & Sig. (p) & 0.616 & 0.569 & 0.561 & 0.549 & 0.913 & 0.549 \\
\hline & $n$ & 46 & 46 & 46 & 46 & 46 & 46 \\
\hline \multirow{3}{*}{ Modified rankin score } & Coef. Correl. (r) & 0.042 & -0.027 & -0.043 & -0.138 & 0.273 & -0.138 \\
\hline & Sig. (p) & 0.784 & 0.856 & 0.776 & 0.359 & 0.066 & 0.359 \\
\hline & $\mathrm{n}$ & 46 & 46 & 46 & 46 & 46 & 46 \\
\hline \multirow{3}{*}{$\begin{array}{l}\text { Presence of associated right } \\
\text { side aneurysms other than the } \\
\text { bifurcation of the MCA }\end{array}$} & Coef. Correl. (r) & 0.289 & 0.080 & -0.242 & -0.105 & -0.130 & -0.105 \\
\hline & Sig. (p) & 0.052 & 0.598 & 0.105 & 0.489 & 0.390 & 0.489 \\
\hline & $n$ & 46 & 46 & 46 & 46 & 46 & 46 \\
\hline \multirow{3}{*}{$\begin{array}{l}\text { Presence of associated left } \\
\text { side aneurysms other than the } \\
\text { bifurcation of the MCA }\end{array}$} & Coef. Correl. (r) & -0.036 & 0.099 & 0.042 & -0.066 & -0.082 & -0.066 \\
\hline & Sig. (p) & 0.810 & 0.514 & 0.780 & 0.664 & 0.590 & 0.664 \\
\hline & $n$ & 46 & 46 & 46 & 46 & 46 & 46 \\
\hline \multirow{3}{*}{ Size of the aneurysm } & Coef. Correl. (r) & -0.112 & -0.505 & 0.448 & 0.057 & 0.128 & 0.348 \\
\hline & Sig. (p) & 0.457 & $<0.001$ & 0.002 & 0.705 & 0.395 & 0.018 \\
\hline & $n$ & 46 & 46 & 46 & 46 & 46 & 46 \\
\hline \multirow{3}{*}{ M1 segment $>3 \mathrm{~cm}$} & Coef. Correl. (r) & -0.178 & -0.018 & 0.206 & -0.127 & -0.157 & 0.359 \\
\hline & Sig. (p) & 0.237 & 0.905 & 0.169 & 0.402 & 0.298 & 0.014 \\
\hline & $n$ & 46 & 46 & 46 & 46 & 46 & 46 \\
\hline \multirow{3}{*}{ Trifurcated middle cerebral artery } & Coef. Correl. (r) & -0.079 & 0.038 & 0.004 & 0.268 & -0.092 & -0.074 \\
\hline & Sig. (p) & 0.601 & 0.801 & 0.980 & 0.072 & 0.542 & 0.623 \\
\hline & $n$ & 46 & 46 & 46 & 46 & 46 & 46 \\
\hline \multirow{3}{*}{ Aspect ratio $>1,5$} & Coef. Correl. (r) & -0.051 & -0.163 & 0.283 & -0.047 & -0.149 & 0.171 \\
\hline & Sig. (p) & 0.739 & 0.280 & 0.057 & 0.754 & 0.323 & 0.256 \\
\hline & $n$ & 46 & 46 & 46 & 46 & 46 & 46 \\
\hline
\end{tabular}

*Shaped middle cerebral artery aneurysm. MCA: Middle Cerebral Artery; M1: Segment M1 of Middle Cerebral Artery. 


\section{DISCUSSION}

Intracranial aneurysms occur in diverse shapes and forms. However, shape is an independent factor since the final conformation will depend on the aneurysm wall's outer molecular composition and the influence of external factors such as underlying systemic hypertension, history of tobacco abuse, as well as local environmental changes such as shear stress forces and hemodynamic characteristics of such ${ }^{2}$. In reality, according to classic studies by Pierre Lasjaunias and others, molecular wall weakening was likely the most important factor linked to aneurysm formation, explaining multiple aneurysms, and mirror aneurysmal formation in humans. Several inheritable connective tissue disorders have been associated with intracranial aneurysms, such as autosomal-dominant polycystic kidney disease, Ehlers Danlos type 4 syndrome and neurofibromatosis type $1^{3,4,5}$. Despite the knowledge of several syndromic features related to aneurysm formation, the true incidence of these alterations in the population is still unknown. One feasible hypothesis is that the genetic disorders related to aneurysm formation remain undiagnosed, because there is a high index of phenotypical expression, and a complete genetic evaluation is lacking in most cases. The understanding of the true pathobiology of the aneurysm formation is essential in developing strategies for the prevention, diagnosis and treatment of such a treacherous disease.

It is also well known that hemodynamic alterations within the internal cerebral artery, from either anatomic/pathologic hypoplastic segments or iatrogenic occlusion, can directly influence the development of intracranial aneurysms $s^{6,7}$. There is also evidence that de novo aneurysms can arise in the anterior communicating artery or contralateral internal cerebral artery after coiling or clipping of intracranial aneurysms ${ }^{8,9}$. This imbalance between local hemodynamic stress and arterial wall strength makes the arterial wall prone to aneurysm formation, and factors such as the above-mentioned make them prone to rupture. There is some evidence showing that biologic changes at the cellular and molecular levels are related to a higher risk of aneurysm formation ${ }^{10,11,12,13}$. Human samples and animal models show a common pathway for the intracranial birth, progression and subsequent rupture, initiating with the endothelial dysfunction and progressing with inflammation and degenerative changes that culminate with aneurysm rupture ${ }^{11,12}$.

Aneurysm rupture risk is related to the aneurysm morphology, in association with the aneurysm location and local hemodynamic factors rather than size, as initially suggested $^{14,15,16,17}$. There are significant controversial points in the management of intracranial aneurysms and, in most circumstances, small aneurysms will rupture in a not-insignificant proportion in clinical practice and accurate answers on their treatment indications are lacking.

Since the middle cerebral artery is embryological and phylogenetically diverse and has different developmental ages, anatomical variations and genetic compositions, aneurysm formation processes in this topography are typically complex, frequently involving multiple branches and sometimes having an unfavorable neck for unassisted endovascular embolization, which can make microsurgical clipping hazardous. The challenge of a perfect arterial dissection and identification of crucial branches that cannot be sacrificed during clipping makes the microsurgical procedure even more difficult. In some instances, utilization of the STA-MCA bypass techniques ${ }^{18}$, ultrasound flow probe measurement of branch flow patency ${ }^{192,2,21}$, and adequate neurosurgical technique with proper temporary clipping application, will decrease morbidity and mortality associated with microsurgical approaches for middle cerebral artery aneurysms. The improvement in imaging technology permits adequate study of the vascular anatomy of the patients, directions of the vessels and detailed assessment of the three-dimensional morphology expected intra-operatively ${ }^{22}$.

As presented in our study, middle cerebral artery aneurysms can present with diverse morphological features. Our study showed that neither pre-operative rupture nor intraoperative rupture are associated with a specific morphology in aneurysms of the bifurcation of the middle cerebral artery, but further studies with larger series are needed to reach a firm conclusion on this topic. Ruptured aneurysms are associated with higher morbidity and mortality and treatment after rupture will significantly affect outcome, since the chances of clinical cerebral vasospasm becomes higher, increasing the long-term morbidity and mortality associated with those $\mathrm{e}^{23,24,25,26,27,28}$. In addition, aneurysm dissection after a rupture can be challenging, and correct neck identification may not be optimal. Survival and outcomes will be directly linked to the postoperative neurological critical care.

In conclusion, we noticed that morphology does not seem to be directly related to an increased rate of pre-operative or intra-operative rupture when dealing with middle cerebral artery bifurcation aneurysms. Pre- or intra-operative ruptures were more likely to be related to higher morbidity and mortality, albeit directly dependent on how aggressive the neurological critical care was carried out after surgery. Larger multicenter case series and uniform treatment paradigms will likely correlate with safety of microsurgical treatments, particularly when endovascular treatment is not indicated or with ruptured aneurysms that cannot be coiled unassisted, i.e., without stent or balloon utilization. Medical treatments that inhibit the inflammatory cascade are likely to prevent progression and rupture of aneurysms ${ }^{12}$. Statins and anti-inflammatory therapies using aspirin will permit, in the longer term, one more step in the comprehension of aneurysm wall formation and rupture. Further studies are needed to correlate the shape of the aneurysms and the risk of rupture and the feasibility of microsurgical clipping without raising the morbidity/mortality of the microsurgical procedure. All effort should be made to stabilize the progression of the disease and diminish the risks of rupture and the morbidity and mortality related to the surgical procedures of intracranial aneurysms. 
1. Heros RC, Fritsch MJ. Surgical management of middle cerebral artery aneurysms. Neurosurgery. 2001;48(4):780-5.

2. Krings T, Lasjaunias PL, Geibprasert S, Pereira V, Hans FJ. The aneurysmal wall. The key to a subclassification of intracranial arterial aneurysm vasculopathies? Interv Neuroradiol. 2008;14(1 Suppl):39-47. https://doi.org/10.1177/15910199080140S107

3. Gibbs GF, Huston J 3rd, Qian Q, Kubly V, Harris PC, Brown RD Jr et al. Follow-up of intracranial aneurysms in autosomal-dominant polycystic kidney disease. Kidney Int. 2004;65(5):1621-7. https://doi.org/10.1111/j.1523-1755.2004.00572.x

4. Pepin M, Schwarze U, Superti-Furga A, Byers PH: Clinical and genetic features of Ehlers-Danlos syndrome type IV, the vascular type. N Engl J Med. 2000;342(10):673-80. https://doi.org/10.1056/NEJM200003093421001

5. Ronkainen A, Hernesniemi J, Puranen M, Niemitukia L, Vanninen R, Ryynänen M et al. Familial intracranial aneurysms. Lancet. 1997;349(9049):380-4. https://doi.org/10.1016/S0140-6736(97)80009-8

6. Gast AN, Sprengers ME, Rooij WJ, Lavini C, Sluzewski M, Majoie CB. Long-term 3T MR angiography follow-up after therapeutic occlusion of the internal carotid artery to detect possible de novo aneurysm formation. AJNR Am J Neuroradiol. 2007;28(3):508-10.

7. Jou LD, Lee DH, Morsi H, Mawad ME. Wall shear stress on ruptured and unruptured intracranial aneurysms at the internal carotid artery. AJNR Am J Neuroradiol. 2008;29(9):1761-7. https://doi.org/10.3174/ajnr.A1180

8. Ferns SP, Sprengers ME, Rooij WJ, Berg R, Velthuis BK, Kort GA et al. De novo aneurysm formation and growth of untreated aneurysms: a 5-year MRA follow-up in a large cohort of patients with coiled aneurysms and review of the literature. Stroke. 2011;42(2):313-8. https://doi.org/10.1161/STROKEAHA.110.591594

9. Miller CA, Hill SA, Hunt WE. “De novo” aneurysms: a clinical review. Surg Neurol.1985;24(2):173-80.https://doi.org/10.1016/0090-3019(85)90181-8

10. Bourcier R, Redon R, Desal H. Genetic investigations on intracranial aneurysm: update and perspectives. J Neuroradiol. 2015;42(2):67-71. https://doi.org/10.1016/j.neurad.2015.01.002

11. Fukuda M, Aoki T. Molecular basis for intracranial aneurysm formation. Acta Neurochir Suppl. 2015;20:13-5. https://doi.org/10.1007/978-3-319-04981-6_2.

12. Kataoka H. Molecular mechanisms of the formation and progression of intracranial aneurysms. Neurol Med Chir (Tokyo). 2015;55(3):214-29. https://doi.org/10.2176/nmc.ra.2014-0337

13. Sathyan S, Koshy LV, Balan S, Easwer HV, Premkumar S, Nair S et al. Association of Versican (VCAN) gene polymorphisms rs251124 and rs2287926 (G428D), with intracranial aneurysm. Meta Gene. 2014;2:651-60. https://doi.org/10.1016/j.mgene.2014.07.001

14. Ausman JI, Roitberg B. A response from the ISUIA. Surg Neurol. 1999;52(4):428-30.

15. Marshman LA, Aspoas AR, Rai MS, Chawda SJ. The implications of ISAT and ISUIA for the management of cerebral aneurysms during pregnancy. Neurosurg Rev. 2007;30(3):177-80. https://doi.org/10.1007/s10143-007-0074-8

16. Raymond J, Guillemin F, Proust F, Molyneux AJ, Fox AJ, Claiborne JS et al. Unruptured intracranial aneurysms: a critical review of the international study of unruptured intracranial aneurysms (ISUIA) and of appropriate methods to address the clinical problem. Interv Neuroradiol. 2008;14(1):85-96. https://doi.org/10.1177/159101990801400111

17. Raymond J, Nguyen T, Chagnon M, Gevry G. Bsc, the trial on endovascular aneurysm management collaborative g: unruptured intracranial aneurysms. opinions of experts in endovascular treatment are coherent,weighted in favour of treatment, and incompatible with ISUIA. Interv Neuroradiol . 2007;13(3):225-37. https://doi.org/10.1177/159101990701300302

18. Inoue T, Yoshida H, Tamura A, Saito I. Clipping and STA-MCA bypass for unrupturedAcomA aneurysm associated with unilateral MCA occlusion. Neurosurg Focus. 2015;38:Video2. https://doi.org/10.3171/2015.V1.FOCUS14469

19. Amin-Hanjani S, Meglio G, Gatto R, Bauer A, Charbel FT. The utility of intraoperative blood flow measurement during aneurysm surgery using an ultrasonic perivascular flow probe. Neurosurgery. 2008;62(6 Suppl 3):1346-53. https://doi.org/10.1227/01.NEU.0000209339.47929.34

20. Charbel FT, Hoffman WE, Misra M, Hannigan K, Ausman JI. Role of a perivascular ultrasonic micro-flow probe in aneurysm surgery. Neurol Med Chir (Tokyo). 1998;38 Suppl:35-8. https://doi.org/10.2176/nmc.38.suppl_35

21. Fagundes-Pereyra WJ, Hoffman WE, Misra M, Charbel FT. Clip readjustment in aneurysm surgery after flow evaluation using the ultrasonic perivascular probe: case report. Arq Neuropsiquiatr. 2005;63(2A):339-44. https://doi.org/10.1590/S0004-282X2005000200028

22. Zurada A, Gielecki J, Tubbs RS, Loukas M, Maksymowicz W, Cohen-Gadol AA et al. Three-dimensional morphometrical analysis of the M1 segment of the middle cerebral artery: potential clinical and neurosurgical implications. Clin Anat. 2011;24(1):34-46. https://doi.org/10.1002/ca.21051

23. Carter BS. ISAT subgroups: is aneurysm clipping better for some patients? Neurosurgery. 2008;63(6):14. https://doi.org/10.1227/01.NEU.0000313633.74773.E7

24. Maurice-Williams RS. Aneurysm surgery after the International Subarachnoid Aneurysm Trial (ISAT). J Neurol Neurosurg Psychiatry. 2004;75(6):807-8. https://doi.org/10.1136/jnnp.2004.036962

25. Molyneux A, Kerr R, Stratton I, Sandercock P, Clarke M, Shrimpton J et al. International Subarachnoid Aneurysm Trial (ISAT) of neurosurgical clipping versus endovascular coiling in 2143 patients with ruptured intracranial aneurysms: a randomized trial.J Stroke Cerebrovasc Dis. 2002;11(6):304-14. https://doi.org/10.1053/jscd.2002.130390

26. Molyneux A, Kerr R, Stratton I, Sandercock P, Clarke M, Shrimpton $J$ et al. International Subarachnoid Aneurysm Trial (ISAT) of neurosurgical clipping versus endovascular coiling in 2143 patients with ruptured intracranial aneurysms: a randomised trial. Lancet. 2002;360(9342):1267-74. https://doi.org/10.1016/S0140-6736(02)11314-6

27. Risselada R, Lingsma HF, Bauer-Mehren A, Friedrich CM, Molyneux AJ, Kerr RS et al. Prediction of 60 day case-fatality after aneurysmal subarachnoid haemorrhage: results from the International Subarachnoid Aneurysm Trial (ISAT). Eur J Epidemiol. 2010;25(4):261-6. https://doi.org/10.1007/s10654-010-9432-x

28. Sellar R, Molyneux A. ISAT: The International Subarachnoid Aneurysm Trail. Lessons and Update. Interv Neuroradiol. 2008;14(1 Suppl):50-1. https://doi.org/10.1177/15910199080140\$109 